\title{
Tsafon
}

Revue d'études juives du Nord

$77 \mid 2019$

Contribution à l'histoire des traductions juives de la Bible hébraïque

\section{L’Histoire, « L'antisémitisme en France »}

\section{Danielle Delmaire}

\section{(2) OpenEdition}

\section{Journals}

Édition électronique

URL : https://journals.openedition.org/tsafon/2170

DOI : $10.4000 /$ tsafon. 2170

ISSN : 2609-6420

Éditeur

Association Jean-Marie Delmaire

\section{Édition imprimée}

Date de publication : 1 septembre 2019

Pagination : 181-183

ISSN : 1149-6630

\section{Référence électronique}

Danielle Delmaire, «L'Histoire, «L'antisémitisme en France » ", Tsafon [En ligne], 77 | 2019, mis en ligne le 09 septembre 2019, consulté le 24 juin 2021. URL : http://journals.openedition.org/tsafon/2170 ; DOI : https://doi.org/10.4000/tsafon.2170 


\section{À travers les livres et un documentaire......}

Coutel Charles, Leduc Christophe, Rota Olivier (études réunies sous la direction de), La culture du dialogue dans les relations inter-religieuses - Définitions, controverses, devenir, Paris, éd. Parole et Silence, janvier 2019, 188 p., $18 €$.

Le dialogue est devenu une culture depuis la fin des années soixante, dont la pratique n'est pas définitivement acquise. C'est particulièrement vrai du dialogue inter-religieux qui peut susciter de fausses interprétations et mener à la désillusion s'il n'est pas correctement défini. Aussi la réflexion proposée dans ce livre codirigé par Charles Coutel, Christophe Leduc et Olivier Rota, de l'Université d'Artois, est-elle intéressante dans la mesure où elle balise l'émergence et le développement du dialogue interreligieux, en précise les enjeux et en souligne les caractères essentiels à la lumière de ses échecs mais aussi de ses réussites.

Ce volume réunit les contributions des onze universitaires qui ont participé à la journée d'étude sur ce thème organisée par l'Institut d'Étude des Faits Religieux (IEFR) de l'Université d'Artois. Dans une introduction d'une dizaine de pages, Charles Coutel par ailleurs auteur de l'article consacré à la définition des termes -, Christophe Leduc et Olivier Rota rappellent très opportunément et très clairement les conditions historiques qui ont imposé « la culture du dialogue » à l'Église catholique après la Seconde Guerre mondiale : celui-ci prit tout son sens lors du concile du Vatican II, réuni à l'initiative du pape Jean XXIII et achevé sous le pontificat de Paul VI, son successeur.

Ce dialogue a ses précurseurs, à l'image des Ancelles, un groupe d'une trentaine de personnes. De 1927 à 1964, ces religieuses, d'abord indépendantes puis rattachées à la congrégation des sœurs de Notre-Dame de Sion avant de recouvrer leur autonomie sous le nom de Pax nostra, se consacrent, rappelle Paule Marx, docteure de l'Université Paris IV-Sorbonne, au «dialogue de vie » avec les juifs : "Les Ancelles s'impliquent, avant la mise en place des grands textes magistraux, dans une action sociale qui va prendre avec le temps les aspects d'un dialogue avec le judaïsme ».

Comment l'Église peut-elle être au monde sans s'y perdre, remplir sa mission évangélisatrice sans renoncer à ses dogmes ? Le dialogue est la voie qu'elle choisit non sans à-coups. Longuement mûrie et discutée, la réflexion aboutit en définitive à la rédaction de documents de référence, analysés par Olivier Rota, de l'Université d'Artois, et Marie-Hélène Robert, de l'Université catholique de Lyon. Le premier jalonne l'émergence du dialogue dans le catholicisme anglais et distingue ainsi l'œcuménisme du dialogue inter-religieux. La seconde centre son intervention sur la notion théologique du dialogue dans l'encyclique Ecclesiam suam. 
Successeur de Jean XXIII, Paul VI s'inscrit pleinement dans la démarche de son prédécesseur et publie l'encyclique Ecclesiam suam le 6 août 1964 et la déclaration conciliaire Nostra aetate est promulguée le 28 octobre 1965. Celle-ci fonde effectivement ce qui devient le dialogue inter-religieux, autrement dit les relations que l'Église souhaite instaurer avec les religions non chrétiennes, à commencer par le judaïsme.

La puissance publique elle-même n'est pas restée en marge de cette évolution. Elle s'est intéressée au dialogue inter-religieux au risque de l'instrumentaliser et de le fausser en le réduisant à une pratique et un calcul politiques. Dans une partie intitulée « L'État et le dialogue entre religions : études de cas », trois communications retiennent l'attention. Éliezer Schilt, docteur de l'Université du Néguev, retrace la brève histoire du CERIM, Comité pour l'Entente Religieuse en Israël et dans le Monde réuni à l'initiative d'intellectuels et de religieux israéliens, dont le promoteur André Chouraqui, et soutenu par l'État d'Israël de sa fondation le 29 décembre 1958 à son extinction progressive en 1965. De son côté, Danielle Delmaire, de l'Université de Lille, témoigne d'une intégration réussie avec l'assentiment des autorités que représente l'œuvre SaintJacques. Elle fut, est et demeure « un lieu de dialogue entre catholiques hébréophones et juifs dans la société israélienne » pour reprendre le titre de son article. Écrit par Hassan Diab El Harake, de l'Université de Lille, le dernier article de cette partie est consacré à l'imam chiite Moussa Sadr, «l'une des figures emblématiques du dialogue islamochrétien » et personnalité religieuse impliquée dans les luttes sociales libanaises au service de l'homme, disparue en Libye en 1978.

Pour autant, le dialogue suscite encore des réticences, des oppositions qui font l'objet de la quatrième partie. Doctorante à l'EPHE, Carole Chrétien rend compte de l'hostilité des traditionalistes catholiques au dialogue inter-religieux en analysant le blog «Contra Nostra aetate » et la Lettre Serviam. Jean-François Petit, de l'Institut catholique de Paris, «s'interroge sur la menace d'obsolescence qui pèse aujourd'hui, de manière paradoxale, sur les formes de dialogue inter-religieux ».

Des « Paroles d'acteurs » concluent cet ouvrage. Attaché à bien cerner la réalité pour éviter toute désillusion, le père Jean Druel, directeur de l'Institut dominicain d'études orientales du Caire, livre une réflexion intéressante sur le dialogue islamo-chrétien en dissociant les différents niveaux d'échanges que peuvent avoir les catholiques et les musulmans. De son côté, Jean-François Bensahel, président de la synagogue de la rue Copernic, plaide avec éloquence pour le contact, l'échange et la connaissance entre les religions, au rebours de la violence et de l'exclusivisme pour conclure à propos de ce dialogue : «Il n'y a pas d'autre alternative à la violence. Il n'y a pas d'autre alternative à l'établissement des temps messianiques ».

Emmanuel Persyn

Decout Maxime, Levy Nurit et Tauber Michèle (sous la direction de), Les appropriations $d u$ discours antisémite. Comportements mimétiques et détournements carnavalesques, Lormont, éditions Le Bord de l'eau, 2018, 220 p., $24 €$.

La littérature française possède ses écrivains antisémites bien connus. Ce qui est moins connu, c'est la façon dont les écrivains juifs ont pu reprendre leurs stéréotypes, se risquant à l'ambiguïté. C'est à explorer cette littérature particulière que s'attellent les contributeurs du présent ouvrage.

Maxime Decout, faisant un large tour d'horizon littéraire, tente d'y débusquer la haine de soi ou un rapport problématique à l'identité juive. Distinguant renversement positif du stéréotype (chez Cohen ou Gary par exemple) et ambivalence du réemploi (chez 
Proust ou Nemirovsky), il souligne que le problème ne se pose plus de la même manière après la Deuxième Guerre mondiale.

L'ambiguïté de Heine, étudiée par Andrée Lerousseau, passe par la satire et le burlesque, avec des personnages cherchant en vain à s'assimiler en singeant les gentils, sans parvenir à masquer les traits distinctifs du Juif, sans renoncer au Witz (esprit humoristique). Adhésion ou autodérision? Amour ou haine ? Cela reste indécidable. De la même façon, l'ambivalence de Proust (analysée par Béatrice Athias) s'exprime à travers des personnages juifs très différents : Swann, symbole de réussite remarquable, osant s'affirmer farouchement dreyfusard, et son double négatif, Bloch, tentant maladroitement de faire oublier sa judéité jusqu'à changer de nom, tandis que l'actrice Rachel déclame du La Fontaine avec une sensualité tout orientale. L'œuvre est émaillée de propos antisémites, et il est difficile de faire la part chez Proust de la défiance et de l'allégeance au discours antisémite. Le cas d'Irène Némirovsky, examiné par Amotz Giladi, semble tout à fait particulier, car elle véhicule dans son œuvre les principaux thèmes antisémites (association Juif-capitalisme, Juif mentalement et physiquement déformé, portant préjudice aux travailleurs du même domaine) sans renier sa judéité. Sa légitimité gagnée par cette attitude paradoxale, elle la perdra dès 1940, et définitivement en 1942, à Auschwitz.

Pour certains écrivains, c'est une haine de soi que véhicule leur œuvre. Ainsi, selon Louis-Albert Revah, Emmanuel Berl hérite de sa famille maternelle une certaine honte d'être juif et fera sien le discours antisémite suivant lequel les Juifs ne peuvent être tout à fait français, allant jusqu'à collaborer avec Pétain et exprimer dans son œuvre la crainte qu'avec «l'immigration de déchet », culture et civilisation ne se perdent. Notons cependant qu'après 1967, il devient un ardent sioniste.

Prenant conscience de son identité juive avec la répression antisémite du régime de Vichy, Bernard Franck, dont Nelly Wolf présente le cas, reprend les thèmes antisémites (Juif déicide, dominateur, violeur) dans la plupart de ses œuvres sous des procédés littéraires lui permettant une double attitude: la bouffonnerie, le retournement de situation (il applique aux antisémites les stéréotypes négatifs qu'ils véhiculent), et la mythomanie. Puis c'est un roman très particulier de Romain Gary qui est analysé par Yves Baudelle : La Danse de Gengis Cohn (1966), livre tout d'ironie qui gagnera les foudres d'Adorno. Un rescapé des camps devient le dibbouk de son tortionnaire nazi et, paradoxalement, c'est l'intrus qui se laisse contaminer par les idées de son hôte. Par l'humour, qui pour Gary est « une défense contre le malheur », il entend dénoncer deux impostures : celle de la culture occidentale impuissante à empêcher l'holocauste et celle de la dénazification de l'Allemagne.

Un autre roman, Jacob le menteur (1969), de Jurek Becker, s'approprie l'idée que les Juifs sont des menteurs : ainsi, un Juif fait croire à ses amis du ghetto que les troupes russes arrivent, ce qui leur permet de reprendre espoir et ainsi de résister à la dureté de la vie dans le ghetto. Le héros est ainsi un « imposteur altruiste » qui ment pour sauver les autres et leur rendre la maîtrise de leur destin (même si tout se termine par la déportation).

Un rapprochement intéressant est opéré par Nurit Levy entre Doubrovsky et Céline dont l'ambiguïté du discours antisémite est repérée. Doubrovsky reprend les comparaisons animales pour décrire les Juifs et se les attribue à lui-même. Comme Céline, il est à la recherche d'une écriture «pseudo-orale », celle même dont Céline a dit qu'elle n'était pas accessible aux Juifs, incapables d'émotion spontanée. Dans une forme de masochisme, Doubrovsky se culpabilise d'avoir survécu et reprend à son compte les insultes proférées par Céline à l'encontre des "aryens baisés par les Juifs ». C'est un autre genre d'obsession, entre oubli et mémoire, qui tenaille la génération d'après, comme celle de Marc Weitzmann, journaliste connu dont l'œuvre littéraire n'échappe pas à l'ambiguïté selon Elena Quaglia. Il est tiraillé entre l'inquiétude identitaire et la 
critique féroce des faux semblants philosémites et veut revendiquer une identité consciente de ses contradictions, en mettant en scène toutes les problématiques liées à la judéité contemporaine.

C'est l'obsession sexuelle manifeste du Juif chez Philip Roth qu'étudie Steven Sampson. Dans ses différents romans, il dégage les thèmes récurrents de la désacralisation de la religion, du sexe et des renversements tortionnaire-persécuteur et de la violence en prenant comme point de départ le film de Polanski, « Rosemary's baby ».

Michèle Tauber se penche, elle, sur le gros roman de David Grossman, Voir ci-dessous, amour, dans lequel la Shoah est une occasion de réfléchir sur l'homme en général en présentant les répercussions de la Shoah sur la vie d'un sabra. À la manière d'un conte à la Shéhérazade, les limites temporelles et spatiales disparaissent, mêlant auto-ironie, thème du dibbouk et désacralisation. C'est aussi à un renversement saisissant que nous invite Bruno Chaouat avec Le transport de A.H. (1981), de Georges Steiner, roman didactique sur l'hitlérisme et l'«antisémitisme rédempteur». L'auteur met en scène l'argumentaire d'Adolf Hitler, transporté en Amazonie, se présentant comme l'instrument de la logique juive elle-même et se montrant comme le véritable messie. Et Steiner fait le parallèle entre l'élimination des Juifs par les nazis et celle de la forêt amazonienne par l'Occident.

Le dernier article, de Vincent Lowy, est consacré au cinéaste Jean Renoir tel qu'il est présenté dans le film «Voyage à travers le cinéma français » de Bertrand Tavernier. Et de se demander comment ce cinéaste populaire, qui avant la guerre condamnait l'antisémitisme, avait pu se compromettre avec le régime de Vichy et regretter dans une lettre qu'il y ait trop de producteurs israélites, comme pour confirmer la parole de Gabin : «Renoir comme metteur en scène, un génie. Comme homme, une pute ».

Françoise Marti

Gross John, Shylock et son destin. De Shakespeare à la Shoah, Paris, éditions Rue d'Ulm, coll. Aesthetica, 2018, 384 p., $26 €$.

Critique théâtral d'envergure, John Gross (1935-2011) réalise avec cet ouvrage une véritable somme sur le protagoniste du Marchand de Venise, depuis la genèse du personnage de Shakespeare, en passant par ses interprétations et les mises en scènes successives de la pièce jusqu'aux divers avatars de Shylock dans la littérature.

Examinant les sources de l'œuvre, Gross mentionne Le Juif de Malte de Christopher Marlowe (1589) et l'origine légendaire de la « livre de chair» exigée dans le contrat. Si le personnage du financier n'est pas étrange sous la plume de Shakespeare (les personnages d'usuriers sont très présents dans le théâtre de cette époque), il est plus surprenant de le voir prendre comme protagoniste un Juif étant donné leur faible nombre dans la société anglaise à cette époque même si l'action se déroule à Venise. Doté d'un nom original, Shylock, dont l'origine n'a pas été trouvée malgré les recherches, le personnage échappe également aux stéréotypes les plus grossiers, doté d'une fine psychologie, aimant sa fille qui représente « la fille vertueuse du méchant Juif ». Lui comme Portia revendiquent la Justice, mais si Shylock reste intransigeant sur la lettre et revendique la légitimité de son dû, Portia en affirme l'esprit, appelle Shylock à l'esprit de miséricorde auquel il refuse d'accéder. Construite sur l'opposition entre les Chrétiens Antonio et Portia, d'une part, et le Juif Shylock, l'étranger, d'autre part, aucun personnage n'est cependant réduit à un stéréotype, tous les personnages de la pièce sont complexes, ce qui autorisera toutes les interprétations au gré des metteurs en scène au fil des siècles.

Gross examine ensuite de manière exhaustive les représentations de la pièce de 1600 à 1939, représentations innombrables, car depuis sa rédaction, exceptée une éclipse au 
$\mathrm{XVII}^{\mathrm{e}}$ siècle (1642-1701), Le Marchand de Venise n'a jamais cessé d'être mis en scène de diverses façons, allant du comique au tragique, et en divers lieux, de l'Angleterre aux États-Unis ou au Japon. Les représentations de la première moitié du XVIII ${ }^{\mathrm{e}}$ siècle peignent un Shylock féroce, les spectateurs de l'époque considérant naturelle une certaine hostilité envers les Juifs. Dès la fin du siècle, apparaissent des écrits prenant la défense du personnage, arguant que la conduite de Shylock était le résultat des mauvais traitements qui lui étaient infligés. Mais c'est au XIX ${ }^{\mathrm{e}}$ siècle que le personnage connaît une véritable réhabilitation. L'interprétation innovante d'Edmund Kean (de 1814 à 1833), demeurée légendaire, parvient à travers le sordide à insuffler au personnage humanité et dignité. Les critiques également changent de lecture : Shylock est pris en pitié, les chrétiens sont condamnés (Hazlitt), on va même jusqu'à soutenir que Shakespeare a su s'affranchir des préjugés haineux de son époque (Hunt), et au milieu $\mathrm{du}$ siècle son personnage est presque émouvant. Henry Irving, à la fin du siècle, interprète un Shylock victime, tandis que les chrétiens deviennent de cruels oppresseurs, faisant de la pièce un plaidoyer pour la tolérance. Certains critiques cependant, en particulier aux États-Unis au début du $\mathrm{XX}^{\mathrm{e}}$ siècle, insistent pour resituer Le Marchand de Venise dans son contexte shakespearien et estiment que l'interprétation romantique est un contresens. L'entre-deux-guerres voit toujours de très nombreuses représentations de la pièce, y compris en yiddish, et même si l'on retrouve des Shylock inflexibles, le personnage est majoritairement interprété comme un héros tragique. Les événements de 1933 semblent n'avoir aucune incidence sur la compréhension de la pièce. Ce sera bien différent, évidemment, après la guerre.

Dans la troisième partie de son ouvrage, Gross s'attache aux avatars de Shylock dans la littérature, personnage devenu mythique à l'instar de Don Quichotte ou de Tartuffe... L'auteur montre l'attitude ambivalente de certains écrivains (Byron), mais comment Dickens renverse ce mythe avec le personnage de Riah. En même temps, la pièce inspire beaucoup de satires ou de parodies mettant en relation le propos de la pièce avec les événements (Disraeli) et est inscrite aux programmes des écoles au Royaume-Uni et aux États-Unis. En Allemagne, Lessing s'en inspire pour créer Nathan le Sage (1779), en faisant un marchand juif tolérant et vertueux, mais, pour beaucoup de critiques, Shylock demeure un scélérat, tandis que les mises en scène varient du héros féroce à l'homme blessé. En France, Le Marchand de Venise n'est pas joué avant 1830, mais tous connaissent Shylock, le Juif grippe-sous. Sur les planches, très peu de représentations, jusqu'à ce que Firmin Gémier attire les foules en faisant de Shylock un «bouffon vengeur hystérique ». Cependant, la renommée de Shakespeare est mondiale et, à la fin $\mathrm{du} \mathrm{XIX}^{\mathrm{e}}$ siècle, Le Marchand de Venise est traduit en 20 langues. Gross étudie également les réactions des écrivains juifs: Heine, Svevo, Proust; en fait, chacun façonne son propre Shylock, jusqu'au film de Lubitsh (« To be or not to be », 1942) où l'acteur déclame dans les décombres de Varsovie : « Un Juif n'a-t-il pas des yeux ?... ».

Après la parole tranchante de Marx : «L'argent est le dieu jaloux d'Israël», les interprétations marxistes de la pièce ne manqueront pas (Juifs capitalistes), ni non plus les interprétations psychanalytiques après Freud (Juifs vus par les chrétiens comme une menace de castration).

Il semble que le personnage de Shylock ait cristallisé et alimenté le stéréotype antisémite: il n'est pas d'écrit antisémite qui ne fasse référence à Shylock. Sous le régime nazi, en Allemagne, Le Marchand de Venise connaît une popularité inégalée, privilégiant une approche venimeuse. Après la Shoah, la question devient épineuse. Dans l'immédiat après-guerre, Shylock est encore un personnage odieux. Ce n'est qu'à partir des années soixante que les metteurs en scène s'apercevront de leur amnésie ou de leur inconscience. Néanmoins, cela n'empêchera pas les interprétations modernistes, ironiques, allégoriques, thématiques ou fantaisistes de la pièce à la fin de $\mathrm{XX}^{\mathrm{e}}$ siècle, 
toutes lectures réductrices auxquelles échappe la complexité des caractères créés par Shakespeare.

Finalement, l'auteur de l'étude estime qu'il est impossible pour un contemporain d'avoir accès à la mentalité d'un chrétien de l'époque élisabéthaine, et qu'il est devenu impossible, après la Shoah, de lire la pièce comme à son origine.

Memmi Albert, Journal de guerre (1939-1943) suivi de Journal d'un travailleur forcé et autres textes de circonstance, Paris, CNRS, fév. 2019, 304 p., $10 €$.

Édité et annoté par Guy Dugas, cet ouvrage réunit plusieurs écrits de l'écrivain et essayiste tunisien Albert Memmi : son Journal de guerre, écrit de 1939 à 1943, le Journal d'un travailleur forcé et, pour terminer, d'autres textes de circonstance. Au terme d'un travail remarquable, Guy Dugas a reconstitué ces articles d'une ou plusieurs pages manuscrites, dont certaines s'avèrent une suite de fragments, voire de très courtes annotations.

Qu'elles soient ou non développées, ces pages précisent l'attitude d'Albert Memmi durant la Seconde Guerre mondiale. Il n'est alors qu'un jeune homme, à peine âgé de 19 ans, davantage préoccupé, écrit Guy Dugas, «...par ses études, ses tenues vestimentaires, la fréquentation de la gent féminine et le militantisme juif que par les événements qui ont commencé à secouer l'Europe ». Le 3 septembre 1939, Albert Memmi écrit ainsi : «Je suis contre la guerre, je tiens nullement à la faire. Je n'irai que si j’y étais obligé ».

Il réfléchit, mûrit, prend néanmoins parti lorsqu'il perçoit clairement la dimension antisémite des événements. Ainsi, le 24 juin 1940, écrit-il : «C'est curieux, après beaucoup d'appels à la modernité, beaucoup de raisonnements que je m'impose, beaucoup de la résignation, où je me dis qu'il vaut mieux accepter, céder, etc., combien je sens en moi rejaillir la révolte ». Une révolte née de ce que «...je porte le poids d'être juif ». Et de préciser un peu plus loin : «Le Juif ne crée pas l'antisémitisme par sa façon de faire ; son existence crée l'antisémitisme ». Au fil des pages de son journal, il dénonce vigoureusement l'antisémitisme des dirigeants allemands, italiens et français. « L'antisémitisme est tellement fort, tellement grandissant que je commence à douter de tout », écrit-il par exemple le 26 mai 1941.

Albert Memmi s'oppose plus nettement encore lorsque les troupes allemandes arrivent en Tunisie. Pourtant, les jeunes Tunisiens ne s'inquiètent pas plus que cela à l'arrivée des troupes allemandes. «Nous n'avions pas réalisé absolument le péril allemand. En réalité, il y avait autre chose : pour tous, les Allemands ne tiendraient pas en Tunisie ; leur séjour ne durerait que quelques jours », écrit Albert Memmi dans un article intitulé « La Question des camps de travail en Tunisie », qui reprend une constatation émise à plusieurs reprises ailleurs dans l'ouvrage.

Cruelle désillusion! L'armée allemande occupe la Tunisie durant sept mois. Sept mois durant lesquels le régime nazi s'en prend à la communauté juive comme il l'a fait dans les autres pays passés sous sa botte. Il est à peine installé qu'il exige, le 6 décembre 1942, des responsables de la communauté juive une liste nominative de mille personnes puis de trois mille... Quelques jours plus tard, il ordonne une première rafle, avec le concours de la police de Vichy, puis les premiers internements. Dans l'article intitulé "Les Juifs de Tunisie pendant l'occupation allemande », Albert Memmi rappelle avec vigueur « ...cette pénible aventure que certains imbéciles ont baptisée 'collaboration' ». Et d'ajouter : « Nous n'avons de leçon à recevoir de personne ».

En Tunisie comme ailleurs, l'attitude des responsables de la communauté juive soulève «...un certain nombre de problèmes qui, comme tous ceux qui engagent des hommes, 
sont noyés dans des flots de passion », note Albert Memmi. Selon lui, la résistance armée était vaine car condamnée d'avance et le refus de fournir des travailleurs aux autorités allemandes « ... aurait abouti à un pogrom général » sans pour autant mettre fin aux demandes de main-d'œuvre de l'occupant. «En acceptant sans lutte ouverte le service du travail obligatoire, les Juifs ont pu limiter les dégâts ; et c'est au fond ce qui importait car le refus total était une pure chimère et peu habile, malgré les apparences », résume-t-il.

Cette tâche est dévolue au «Comité de recrutement de la main-d'œuvre juive », un organisme qui est distinct des institutions communautaires traditionnelles et qu'Albert Memmi décrit et défend dans deux articles repris dans cet ouvrage, non sans pointer qu' «...il a sauvé de préférence une classe de la population : les fils de notables », ce qui engendre des conflits entre les responsables opportunistes de ce comité et sa section dite bureau de recrutement, " chargé d'un travail qui ne pouvait le faire aimer ». Dans les camps, dès le 15 janvier 1943, «...commença une besogne patiente et tenace de désorganisation qui produisit d'excellents résultats », raconte Albert Memmi puisque «...les délégués de la communauté réussirent à vider presque complètement les camps ".

Et, parce que la communauté juive a fait ce choix, écrit Albert Memmi, s'impose la relève des hommes envoyés dans les camps de travail. Le 20 mars 1943, il part pour le camp d'Aïn Hloua. « C'est le jour où j'ai compris toutes ces données du problème que moi, planqué pendant des mois, j'ai rejoint volontairement les camps de travail». « J'avais l'impression de m'enfoncer dans un gouffre sans fond. [...] Le sentiment de ne plus exister en tant que personnalité distincte, ou plus exactement de n'avoir aucune prise sur les événements », se souvient-il.

Volontaire, Albert Memmi n'en souffre pas moins de la faim, de la douleur physique, du manque d'hygiène, de la fatigue. Et plus que tout des brimades constantes, de l'humiliation, de l'antisémitisme. À la lutte permanente pour la simple survie s'ajoute le combat obstiné pour la dignité, l'illustration du judaïsme. Guy Dugas intitule très justement son introduction «Les trois guerres d'Albert Memmi » : rappelant sa vie dans un camp de travail, son combat contre l'antisémitisme et son engagement contre le colonialisme, il voit dans cet ensemble d'écrits « ...un véritable document historique ».

Emmanuel Persyn

Bienenstock Myriam, Cohen und Rosenzweig. Ihre Auseinandersetzung mit dem deutschen Idealismus, Freiburg/München, Verlag Karl Alber, 2018, 298 pages.

L'essai de Myriam Bienenstock, qui propose ici une version revue et augmentée de son étude parue en français en 2009, répond à la nécessité, formulée par Emil Fackenheim dans son «Épitaphe pour le judaïsme allemand», qu'il y aurait à se pencher sur le «Siècle d'Or» de la philosophie allemande si l'on veut restituer pleinement et maintenir vivant l'héritage de Hermann Cohen (1842-1918) et de celui qui fut son élève, Franz Rosenzweig (1886-1929), l'un et l'autre étant trop souvent appréhendés comme étant essentiellement des penseurs juifs dont l'œuvre puiserait en priorité dans la tradition juive. L'essai s'attache ainsi à analyser d'une part la compréhension que ces philosophes ont de l'idéalisme allemand et d'autre part la façon dont ils débattent entre eux sur la base de cette philosophie, apportant ainsi leur contribution à l'histoire des idées en Allemagne.

L'essai - et ce n'est pas la moindre de ses qualités - est construit avec une remarquable rigueur: après le rappel d'un certain nombre de données biographiques, chaque chapitre, placé sous le signe du questionnement, se déploie en trois parties, permettant à l'auteure à la fois d'analyser la façon dont s'élabore la pensée des deux philosophes 
dans un débat permanent avec l'idéalisme allemand, leur appréhension étant parfois infléchie par la réception de celui-ci chez leurs contemporains (Dilthey ou, pour ce qui concerne Rosenzweig, Meinecke). Cette lecture «orientée» est pour l'auteure l'occasion d'exercer sa propre réflexion critique. Ainsi Myriam Bienenstock se fait-elle à maintes reprises l'avocate de Hegel, lorsqu'elle s'inscrit par exemple en faux contre «l'eschatologisation» de la pensée hégélienne à laquelle procède Rosenzweig sous l'influence d'Ehrenberg, rappelant que la visée ultime de Hegel n'était nullement la Rédemption, mais la liberté politique, et elle n'hésite pas non plus à revisiter l'image de Cohen esquissée par Rosenzweig et reprise par Löwith, tendant à faire également du messianisme de Cohen une eschatologie, le prophétisme et le messianisme étant bien plutôt chez Cohen, soucieux de maintenir à l'horizon un idéal politique socialiste, au fondement d'une « éthique sociale ». L'auteure souligne en outre la lecture quelque peu biaisée que fait Rosenzweig de la philosophie politique de Hegel au prisme de l'enseignement de Meinecke, même si Rosenzweig parvient à se démarquer de son maître. Dans sa critique de l'idéalisme transcendantal dirigée aussi contre Hegel, Rosenzweig semble enfin ignorer la critique émise par le philosophe lui-même à l'encontre de cet idéalisme.

Nourries de leur confrontation avec le christianisme d'une part et l'idéalisme allemand d'autre part, la philosophie de Cohen et la « pensée nouvelle » de Rosenzweig s'édifient en particulier à partir de ces pierres d'achoppement que sont, pour ces penseurs juifs, la tentation de représenter Dieu et l'anthropomorphisme dans l'art, le panthéisme hérité de Spinoza, la notion de médiation ou d'incarnation, la philosophie du moi développée par Fichte et de l'intériorité chère à l'école romantique, la réduction - à l'exception peutêtre de Herder - de la langue au logos ou plus exactement à une logique étrangère à la langue, le mythe et la "mythologisation » de l'histoire dans l'historisme dénoncé par Rosenzweig, etc. Le débat autour du panthéisme responsable de nombre de maux et l'approche critique à la fois de Spinoza et de la lecture de Spinoza effectuée par Schleiermacher et par l'école romantique (imposant à Cohen de rétablir la vérité sur le philosophe) ou par Dilthey, héritier de l'idéalisme, ayant décelé chez Hegel un "panthéisme de l'esprit », occupe une place importante du début à la fin de l'essai et témoigne du souci de contextualisation : à la discussion autour du spinozisme et du panthéisme succèdera assez tardivement chez Cohen la critique - radicalisée chez Lévinas - du Traité théologico-politique entreprise dans le contexte de la montée de l'antisémitisme dont le discours trouvait une source inépuisable dans l'antijudaïsme contenu dans le Traité. C'est également dans ce contexte et celui de la Première Guerre mondiale que Cohen, se faisant le défenseur du judaïsme, rappelle le message relatif à l'amour du prochain ou plus précisément au caractère infondé de toute forme de haine contenus dans la Bible et le Talmud, et que Rosenzweig tente d'élaborer avec son cercle d'amis une " pensée nouvelle » en rupture avec l'hégélianisme (sans voir à l'instar de Meinecke dans la philosophie politique de Hegel une philosophie prônant un État fort au sens où l'entendra Bismarck ou une apologie du nationalisme, Rosenzweig n'en soulignait pas moins qu'avec Hegel s'amorçait une évolution dans cette direction).

Ces pierres d'achoppement sont pour Cohen et Rosenzweig l'occasion d'entamer à partir de leur propre perception de la tradition juive, une réflexion sur l'esthétique (voir le chapitre consacré à l'analyse de l'ironie chez Cohen et le rappel de l'esthétique développée par Rosenzweig dans L'Étoile de la Rédemption), et de proposer un certain nombre de redéfinitions : celle du concept «d'autonomie » chez Cohen, une autonomie non plus comprise comme détermination de soi par le soi (la «Sebstbestimmung " fichtéenne) mais fondée sur une «corrélation» entre Dieu et l'homme, entre loi et devoir, et qui, en dépit de l'extériorité maintenue de la loi, n'est pas sans présenter une certaine parenté avec la conception de Kant selon laquelle ce n'est pas le concept de liberté qui fonde l'impératif catégorique, mais l'inverse; ou celle de l'esprit chez 
Rosenzweig et d'une science de l'esprit qui s'édifie sur l'analyse de la langue dont la base est le «Tu», sur cette «sphère» dont Rosenzweig déplore l'absence dans l'idéalisme allemand et même chez Schelling, philosophe qu'il admire. Autant de considérations qui déboucheront sur une philosophie de l'interpellation et de la responsabilité qui culminera chez Lévinas.

Le dernier chapitre qui s'applique d'ailleurs à dégager les traces de Cohen et de Rosenzweig dans l'œuvre de Lévinas, est particulièrement stimulant. Outre une analyse passionnante des écrits de ce philosophe, l'auteure y fait la démonstration que la pensée juive contemporaine ne peut s'appréhender sérieusement que dans une prise en compte de l'héritage de ce judaïsme allemand ancré dans une réflexion autour des deux traditions, juive et allemande, et dont Hermann Cohen et Franz Rosenzweig furent les plus éminents représentants. Le débat semble d'ailleurs se poursuivre, ainsi qu'en témoigne la réponse de Derrida au jugement prononcé par Lévinas sur Heidegger. Il y a quelque chose, dans ce rapport entretenu par les penseurs juifs à la tradition philosophique allemande, qui n'est pas sans rappeler la relation que dit avoir Claude Vigée, poète et essayiste, à Hegel lorsqu'il qualifie celui-ci «d'ennemi intime » : une intimité qui n'impliquerait pas nécessairement une parenté, encore moins une symbiose, mais une fréquentation constante permettant de mieux se situer.

Andrée Lerousseau

Badinter Robert, Idiss, Paris, Fayard, octobre 2018, 227 p., $20 €$.

« J'ai écrit ce livre en hommage à ma grand-mère maternelle, Idiss. Il ne prétend être ni une biographie, ni une étude de la condition des immigrés juifs de l'Empire russe venus à Paris avant 1914. Il est simplement le récit d'une destinée singulière à laquelle $\mathrm{j}$ 'ai souvent rêvé. Puisse-t-il être aussi, au-delà du temps écoulé, un témoignage d'amour de son petit-fils ", telle est l'information que Robert Badinter donne dans la p. 4 de couverture. Et voilà résumé, en quelques lignes, le contenu de ce petit livre que l'auteur dédie à ses petits-enfants, se constituant ainsi le chainon de la lignée familiale, entre une femme, illettrée, immigrée de sa Russie natale vers une France qu'elle croyait libératrice, et de jeunes Français, intégrés dans l'intelligentsia française, cent ans plus tard. Quel ravissement pour le lecteur de pénétrer, par le truchement des souvenirs de l'auteur, dans cette famille remarquable !

Née en 1863, dans un pauvre shtetel de la Russie tsariste où l'antisémitisme sévissait quotidiennement, Idiss n'eut pas la possibilité de fréquenter une école : les études ne concernaient pas les filles. Mais le bon sens ne lui manquait pas, ni le courage pour faire face à l'absence d'un mari qui quitta sa femme et ses deux fils pour servir dans l'armée du tsar ! Cela dura cinq longues années pendant lesquelles le retour semblait de plus en plus improbable. Avec ce retour naquit le troisième enfant du couple : une fille, Chiffra, la mère de Robert Badinter. La vie était si rude que les fils aînés émigrèrent vers la France et incitèrent le reste de la famille à les rejoindre. C'est ainsi qu'Idiss, accompagnée de Chiffra encore adolescente, traversa l'Europe, elle qui n'avait pas quitté son shtetel, en passant par Vienne, capitale de l'Empire des Habsbourg, emplie de luxe et de frivolité étourdissants.

Dans la France d'avant la Première Guerre mondiale, en 1911, Chiffra, devenue Charlotte, bénéficia des bienfaits de l'école républicaine et découvrit avec bonheur la culture française. Hélas, si elle pouvait accéder à l'éducation, à la différence de sa mère Idiss, Charlotte ne poursuivit pas d'études, l'heure n'était pas encore arrivée où les filles pouvaient entreprendre de longues études, regrette Robert Badinter.

$\mathrm{Au}$ lendemain de la guerre, Idiss devint veuve. Elle s'installa alors chez sa fille qui venait d'épouser Simon Badinter, un jeune Bessarabien. Celui-ci était arrivé en France 
avant la Première Guerre mondiale; il participa à la défense de sa nouvelle patrie lorsque celle-ci se trouva agressée par l'Allemagne. L'entre-deux-guerres fut la période heureuse de la famille. Idiss vivait chez sa fille et son gendre, non loin de ses deux fils mariés eux aussi. Le cocon familial était chaleureux, Idiss entourait ses petits-fils d'un amour discret mais profond et ceux-ci le lui rendaient bien. Le couple Badinter s'intégra dans la société environnante, s'embourgeoisa et obtint la nationalité française en 1927. Simon Badinter vouait une grande admiration à la France, surtout celle du Front Populaire, celle de Léon Blum qu'il écoutait avec ferveur. Il appréciait les valeurs laïques de cette République qu'il trouvait accueillante.

Et pourtant, les nuages assombrissaient ce bonheur, à partir de 1940. La déception s'abattit sur la famille avec les premières mesures antisémites. Les angoisses réinvestissaient Idiss, comme par le passé lorsqu'elle vivait dans la Russie tsariste. Ce bon sens qui l'habitait depuis si longtemps lui faisait pressentir le pire. Elle le redoutait vivement car elle l'avait connu dans son shtetel. Approchant des quatre-vingts ans, Idiss décéda d'un cancer en 1942. Ce pire lui avait été épargné.

Comme a prévenu l'auteur, nous n'apprenons pas grand-chose sur la vie du shtetel, l'immigration juive venant de Russie et l'amour des immigrés pour la République française ; nous en savons encore moins sur le sort de la famille durant la guerre, sinon qu'un oncle fut déporté. Qu'en était-il des enfants et des petits-enfants d'Idiss après son décès? Le lecteur reste sur sa faim.

Danielle Delmaire

Dermenjian Geneviève, Antijudaïsme et antisémitisme en Algérie coloniale, 18301962, Aix-en-Provence, Presses Universitaires de Provence, $4^{\mathrm{e}}$ trim. 2018, 351 p., $26 €$.

Maître de conférences honoraire à l'ÉSPÉ (École supérieure du professorat et de l'éducation) de l'Université Aix-Marseille, Geneviève Dermenjian est désormais connue pour ses nombreux travaux sur les communautés juives d'Algérie et sur l'antisémitisme qui sévissait dans cette colonie française. Son dernier ouvrage, très complet, reprend et augmente ses connaissances sur l'antijudaïsme et l'antisémitisme dans l'Algérie depuis l'arrivée des Français en 1830 (par la guerre) jusqu'à leur départ en 1962 (toujours par la guerre).

Après une introduction où les termes d'antijudaïsme et antisémitisme sont définis bien distinctement: le premier d'ordre religieux, le second d'ordre racial, économique et social, l'étude se présente en trois parties : l'histoire de l'antisémitisme en Algérie, dès avant même la colonisation, une anthropologique sur les différentes composantes de la population en Algérie coloniale, l'exposé des théories et doctrines antisémites à partir de diverse sources.

La présence juive est bien antérieure à l'arrivée des Français et les juifs sont considérés comme des «indigènes » par les premiers colonisateurs jusqu'à ce que le décret Crémieux (1870) les émancipe et les extrait de l'indigénat pour les intégrer dans la nation française. Leur européanisation commence donc dans le dernier quart du XIX siècle et se trouve accélérée par l'implantation des écoles de l'Alliance israélite universelle. Désormais les juifs sont pris en étau entre les Européens, dont une partie se montre antisémite, et les indigènes musulmans encore influencés par la dhimmitude qui relègue les juifs à un rang inférieur. Or il se trouve d'une part des juifs, établis depuis des siècles en Algérie, très proches des « indigènes » musulmans et d'autre part des juifs sortis de l'indigénat par européanisation ou bien parmi les Européens venus coloniser l'Algérie. Durant la Seconde Guerre mondiale, le décret Crémieux fut aboli par le Gouvernement de Vichy et maintenu quelques mois encore par le gouvernement d'Alger, à la grande déception des nombreux juifs résistants. Dans l'Algérie coloniale, 
les juifs ont donc subi des exactions des deux côtés de la société. Les pogroms qui détruisent les quartiers sont souvent initiés par des Européens mais activement pratiqués par la populace européenne et musulmane.

Dans l'historique de la première partie, Geneviève Dermenjian insiste sur les périodes de grande crise antisémite : durant les années 1890 avec le fort écho, en arrière fond, de l'affaire Dreyfus et pendant lesquelles les émules de Edouard Drumont et Max Régis sont nombreux et violents chez les Européens; puis durant les années 1930 avec le contexte tout aussi violent de la montée du nazisme en Allemagne, voire en Europe et en France. Les grands pogroms, dont celui de Constantine en 1934, se situent durant ces années. La période de la guerre légalise l'antisémitisme et correspond à l'ouverture de camps dans le sud algérien où bien des juifs ont souffert de maltraitance. Quant à la période de la guerre d'indépendance, la situation ne s'améliore guère. Majoritairement solidaires des colons partisans de l'Algérie française, les juifs n'en sont pas moins approchés par le FLN qui leur promet un monde meilleur dans une Algérie musulmane et indépendante. Peu nombreux sont ceux qui y croient.

La seconde partie étudie les différentes populations de l'Algérie coloniale et expose la diversité des milieux sociaux comme la bourgeoisie européenne dans laquelle se fondent peu à peu des familles juives mais aussi musulmanes, même si celles-ci subissent encore le statut de l'indigénat. Toutefois, si l'antisémitisme pénètre dans la majorité des couches sociales aisées ou pauvres, il se trouve toujours des personnalités ou des individus, des voisins qui renoncent à la stigmatisation des juifs. En définitive, il est bien difficile rappelle Geneviève Dermenjian de circonscrire la haine des juifs à tel ou tel groupe social. C'est d'ailleurs ce qu'écrivait le spécialiste de l'histoire de l'Algérie, Benjamin Stora (qui a préfacé l'ouvrage), dans ses souvenirs de Constantine (Les Clés retrouvées. Une enfance juive à Constantine, Stock, 2015) où sa famille, d'origine modeste, fréquentait les voisins arabes. Et pourtant c'est à Constantine que se déroule le plus grand pogrom meurtrier en août 1934.

La troisième et dernière partie présente les théories et les doctrines antisémites. Ce sont les pages les plus originales et les plus novatrices de l'ouvrage. L'auteur scrute les fêtes et les manifestations, analyse le langage et le vocabulaire, surtout celui de la presse et des tracts, pour déceler les mécanismes de la pensée antisémite. Les pages les plus impressionnantes sont celles qui reproduisent et commentent les caricatures qui appartiennent majoritairement aux deux décennies les plus virulentes contre les juifs : 1890 et 1930. Les clichés les plus éculés envahissent les pages de ces publications. Rien d'orignal, en vérité, n'est à retirer de cette propagande antisémite par la caricature. On y trouve les insectes malfaisants, les poux et autres araignées, les mêmes personnalités ciblées comme Dreyfus, Léon Blum ou son ministre Jean Zay.

En fin d'ouvrage, une abondante bibliographie et la liste des sources (archives, mais aussi la presse et la littérature d'époque) renseignent le lecteur.

Si l'ouvrage est à lire, on peut toutefois regretter quelques redondances dues au plan choisi, notamment en ce qui concerne les pogroms.

Heddebaut Monique, Des Tsiganes vers Auschwitz. Le convoi $Z$ du 15 janvier 1944, avant-propos d'Henriette Asséo, Paris, éd. Tirésias, septembre 2018, 323 p., $27 €$.

Les Tsiganes résidant dans les départements du Nord et du Pas-de-Calais furent les seuls, en France, à être déportés. Comme les juifs des mêmes départements, ils furent internés à la caserne Dossin de Malines (Belgique), camp de transit avant la déportation vers Auschwitz. Sur 351 personnes constituant ce convoi, $90 \%$ d'entre eux ne revinrent pas. C'est à l'histoire d'un convoi particulier, le convoi Z du 15 janvier 1944, que 
Monique Heddebaut s'est intéressée pour un master en histoire. Cette recherche excellente et des compléments d'information ont abouti à cet ouvrage. L'auteure est désormais reconnue parmi les spécialistes de la déportation des Tsiganes et du sort de ces derniers dans les camps d'internement en France, durant la Seconde Guerre mondiale. À ce titre, elle a été sollicitée par le Mémorial de la Shoah pour monter, avec d'autres historiens, l'exposition sur les Tsiganes pendant la guerre. Elle avait déjà présenté une partie des ses recherches dans un $n^{\circ}$ hors-série de Tsafon, paru en $2008^{1}$.

La mise à l'écart des Tsiganes dans la société française ne date pas de la Seconde Guerre mondiale. Monique Heddebaut le rappelle avant même d'entamer l'histoire du convoi Z (comme Zigeuners). Depuis bien longtemps, leur vie nomade les rendait suspects et ils étaient des « étrangers de l'intérieur». Une législation anti-tsigane avait été mise en place par la Troisième République. Puis, établis dans les départements du nord de la France rattachés au commandement militaire de Bruxelles dès 1940, ils subirent, tous comme les juifs vivant dans ces mêmes départements, la législation raciale allemande.

C'est à l'automne 1943 que la tragédie commença. Les Tsiganes furent raflés dans leurs campements, sans ménagement. Leurs biens furent confisqués, ils furent spoliés comme les juifs. La population avoisinante se désola mais personne ne vint en aide à cette population marginalisée depuis longtemps.

La vie quotidienne à la caserne Dossin était faite d'humiliation, de vexation et de maltraitance qui attiraient la pitié des autres internés. Les familles étaient regroupées et les enfants nombreux, il y eut même une naissance à Malines.

Le convoi $\mathrm{Z}$ fut acheminé vers Auschwitz. Les femmes y subirent, outre la faim et les maladies, les traitements de stérilisation qui devaient mettre fin à l'existence des Tsiganes: il s'agissait bien d'une "solution finale» et planifiée elle aussi, selon l'auteure. Après quelques mois passés à Auschwitz, des hommes furent transférés à Dora et à Buchenwald où la place manquait, ils survécurent donc sous des tentes. Des femmes furent transférées à Ravensbrück. Les conditions de vie y étaient tout aussi meurtrières et plusieurs Tsiganes y perdirent la vie.

L'accueil au retour des camps, tant de la part de la population que de celle des services chargés de leur venir en aide, ne fut pas à la hauteur des sévices subis. Certes, les rescapés de la déportation raciale furent hospitalisés mais les déportés politiques attiraient toute l'attention des secours, les déportés tsiganes (juifs également) étaient moins honorés, dépourvus de l'auréole de la Résistance! Et les Tsiganes plus particulièrement n'intéressaient pas grand monde. Après la guerre, une législation restrictive avait toujours cours. Faire reconnaître la spoliation d'une roulotte n'était pas facile. Délaissés, les Tsiganes ont replongé dans la mise à l'écart et la stigmatisation maintenues après la guerre.

Monique Heddebaut a travaillé scrupuleusement dans les centres d'archives en Allemagne, en Belgique et en France où elle a pu découvrir des sources encore inexploitées. Ce qui lui a permis de reconstituer la liste des 351 déportés du convoi Z qu'elle reproduit, en fin d'ouvrage. Cette liste est un mémorial comme le sont les deux monuments, sur les lieux des rafles, dont Monique Heddebaut donne la photographie ainsi que la plaque à l'entrée de la caserne Dossin, plaque qui fut apposée après celle rappelant l'internement et la déportation des juifs. 34 déportés revinrent des camps : 21 hommes et 13 femmes qui furent moins nombreuses à survivre ; parmi eux, 3 garçons et 3 filles âgés de moins de 15 ans à leur départ de Malines. Monique Heddebaut donne aussi les noms de ces survivants. Elle a encore retrouvé deux rescapés, Joséphine et Antoine Lagrené, âgés respectivement de 14 et 13 ans à leur départ de Malines et seuls

\footnotetext{
${ }^{1}$ « Persécutions raciales dans le Douaisis pendant la seconde Guerre mondiale, Juifs et
} Tsiganes », Tsafon $\mathrm{n}^{\circ} 4$ hors-série, octobre 2008, 143 p. 
survivants de leur famille d'une trentaine de personnes, qui ont accepté de témoigner. Leurs récits ont apporté d'importants compléments au contenu des archives: les expériences pseudo-médicales subies par Joséphine, les ruses de l'enfant Antoine à Buchenwald. Monique Heddebaut livre là des témoignages inédits.

En outre l'auteure pose, en fin d'ouvrage, les questions pertinentes de vocabulaire : distinguer extermination et génocide, pillage et spoliation.

Enfin est ajoutée, en fin de volume, la liste des fonds d'archives interrogés, tant en France (Archives de Caen, archives nationales, archives de plusieurs départements et de différentes municipalités) qu'en Belgique ou en Allemagne ou encore en Pologne. Les témoignages, autres que ceux de Joséphine et Antoine Lagrené, qui ont été recueillis par l'auteur ou mis par écrit après la guerre sont également listés.

S'y adjoignent une bibliographie abondante, témoignage de l'ampleur du travail de recherche accompli et une courte filmographie.

D.D.

Une réédition :

Wieviorka Annette, Ils étaient juifs, résistants, communistes, Le Grand Livre du Mois, 2018, $421 \mathrm{p}$.

Publié pour la première fois en 1987 chez Denoël et épuisé depuis longtemps, Annette Wieviorka, a décidé de republier Ils étaient juifs, résistants, communistes, en l'augmentant considérablement. Les quelque trente ans qui séparent ces deux éditions, ont permis à l'auteur de disposer des archives accessibles depuis 2005, après le délai légal de 60 ans. Mais surtout, elle a pu exploiter la masse d'archives policières mises à jour depuis le mitan des années 1980 et librement consultables depuis décembre 2015. Elle a ainsi croisé le récit de ceux qui avaient survécu aux rafles et l'a confronté aux archives, ce qui lui a permis de tracer un dessin plus clair, plus assuré, mais également plus complexe de ces femmes et de ces hommes du groupe FTP-MOI [Francs Tireurs et Partisans / Main d'Euvre Immigrée]. Alternant témoignages et analyse, elle a retracé les parcours individuels en leur donnant une densité humaine. De tous ceux qui ont témoigné seuls deux d'entre eux étaient encore parmi nous en 2018. Annette Wieviorka estime qu'il s'agissait là de son « livre le plus personnel » [Le Monde, 27 octobre 2017].

Monique Heddebaut

Un documentaire :

Vilmain Vincent (coordinateur), Judaïsmes européens (1770-1930). Laboratoires des identités partagées, conférence des 5 et 6 mars 2018, enregistrée et parue en ligne: https://judeuro2018.sciencesconf.org. Durée du documentaire : 1 h 16.

À cette conférence ont participé une douzaine de spécialistes de l'histoire des communautés juives européennes, certains d'entre eux plus connaisseurs d'un pays (Angleterre, Italie ou Allemagne etc.) d'autres plus érudits sur les fondements du judaïsme ou les penseurs juifs. Il faut reconnaître un savoir faire et une sagacité remarquables pour trier et associer les différentes prises de paroles des conférenciers afin de restituer un ensemble cohérent et bien équilibré. Le montage a dû occasionner des heures de travail.

La présentation du documentaire donne à comprendre les intentions des organisateurs : « À quelques semaines des élections européennes et dans un contexte de résurgence de 
formes marquées d'antisémitisme en France comme sur l'ensemble du continent, le documentaire Judaïsmes européens (1770-1930). Laboratoires des identités partagées propose une relecture de l'histoire contemporaine de l'idée d'Europe à partir du destin des Juifs sur le continent. Il permet également de s'interroger sur le rapport spécifique que les Juifs ont pu construire à l'Europe comme entité culturelle et comme projet politique, depuis le temps des émancipations jusqu'à l'arrivée du nazisme en Allemagne ». L'auditeur n'est pas déçu, il s'agit bien d'entendre l'avis des historiens sur l'influence des juifs dans la mise en place de l'Europe, celle des Lumières au XVIII ${ }^{\mathrm{e}}$ siècle et des conséquences de celles-ci sur l'existence des juifs, jusqu'à la veille du triomphe de l'antisémitisme en Europe avec l'expansionnisme de l'Allemagne nazie. Quel rôle les juifs ont joué dans l'Europe moderne et quel impact le libéralisme, politique, économique et social, a eu sur les communautés juives ou les destins d'individus? Sujet d'actualité en effet.

L'entrée dans la modernité à la fin du XVIII ${ }^{\mathrm{e}}$ siècle et l'affirmation de la nécessité d'une émancipation bouleverse les communautés où les rabbins voient leur influence diminuer. L'évolution n'est pas générale, elle émerge en Europe occidentale (Allemagne, France, Angleterre) et progresse lentement vers l'est où la Russie reste longtemps rétive à toute émancipation des juifs. En outre, cette libéralisation de la condition des juifs provient des idées révolutionnaires françaises qui, finalement, n'ont pas bonne presse dans les Empires centraux qui connurent l'occupation napoléonienne. La Restauration est une période durant laquelle la régression est générale pour les juifs d'Europe. Il ne faut guère s'étonner, alors, de dénombrer des juifs dans les mouvements libéraux et patriotes favorables à la constitution des nationalités en Europe. Le sionisme n'est-il pas né dans ce contexte et n'est-il pas lui aussi un mouvement de libération nationale?

Est souligné également le rôle des juifs dans le développement des sciences, des techniques ainsi que des arts (musique, littérature, peinture) et de la philosophie, voire même de la linguistique avec les efforts de Zamenhof pour mettre en circulation une langue internationale : l'espéranto. Quelques conférenciers vont jusqu'à évoquer leur rôle de passeur entre le judaïsme et le monde moderne. Passeurs aussi furent les fondateurs de l'Alliance israélite universel qui s'attachèrent à répandre dans le Bassin méditerranéen la culture européenne en général et française en particulier.

À rebours, l'émancipation qui devait libérer les juifs des contraintes antisémites suscite dans les milieux conservateurs, surtout à l'est mais même dans les pays de grande culture des Lumières comme l'Allemagne, un antisémitisme qui ravage les communautés d'Europe centrale et orientale. Ces conditions de vie difficiles n'affaiblirent les sentiments patriotiques des juifs : partout les juifs se sont engagés dans le premier conflit mondial pour défendre leur patrie. Ce qui n'empêchait pas les soupçons de traîtrise qu'une partie de la population nourrissait à leur égard.

Le judaïsme européen est multiple dans les années trente du $\mathrm{XX}^{\mathrm{e}}$ siècle : discret à l'ouest, plus particulier à l'est avec une littérature yiddish brillante. C'est ce monde qui est menacé à la veille de la Seconde Guerre mondiale.

L'écoute de cette conférence est enrichissante même si elle n'apporte pas d'innovation majeure dans nos connaissances. Des documents illustrent les interventions des conférenciers que l'on voit dans une posture un peu trop statique, mais il faut remercier $\mathrm{V}$. Vilmain d'avoir réussi à rassembler une douzaine de collègues pour les inviter à s'exprimer sur tous ces sujets.

Étaient réunis : F. Abecassis, D. Bourel, J. Ehrenfreund, S-A Goldberg, J. Guedj, C. Le Foll, M. Michaud, C. Nicault, S. Nordmann, J-P Schreiber, E. Touboul, V. Vilmain. 


\section{À travers les revues......}

L'Histoire, « L'antisémitisme en France », Les collections de 1'Histoire n 83, avril-juin 2019, $98 \mathrm{p}$.

La parution de ce numéro de la revue L'Histoire, sur un tel sujet, en France dans les premiers mois de 2019, n'est pas anodine. La motivation de la rédaction est évidente lorsqu'on s'attarde sur les illustrations de la couverture : se trouvent l'un au-dessus de l'autre le tableau de Giovanni Canavesio (1492) représentant Jésus devant Caïphe, entouré de juifs au faciès haineux, et le portrait souillé d'une croix gammée de Simone Veil, sur une boîte à lettres du XIII ${ }^{\mathrm{e}}$ arrondissement de Paris, 12 février 2019. L'histoire se répète, l'antisémitisme perdure dans la France du XXI ${ }^{\mathrm{e}}$ siècle depuis au moins le Moyen Âge. Et le contenu de la dizaine de pages qui précède le dossier est tout aussi éloquent: Michel Winock, conseiller de la rédaction, recueille les propos de Pierre Birnbaum, auteur de nombreux livres sur le sujet. Les inquiétudes de l'historien s'expriment dans cette phrase : " $\mathrm{Au} \mathrm{XXI}$ siècle on tue des Juifs en France », non par inadvertance mais bien parce qu'ils sont juifs! La conversation roule sur des événements très récents comme la profanation du cimetière de Quatzenheim (Alsace) en février 2019, la vandalisation du mémorial d'Ilan Halimi le 10 février 2010 ou encore les dérives de quelques "gilets jaunes». "Les vieux mythes ne demandent qu'à renaître » s'alarme P. Birnbaum. Remercions la revue d'informer le lecteur, dans cette même introduction, sur l'initiative de l'historienne M-A Matard-Bonucci qui a créé, en janvier 2019, l'association Alarmer: Association de lutte contre l'antisémitisme et le racisme par la mobilisation de l'enseignement et de la recherche que l'on peut contacter à assocalarmer@gmail.com.

Et précisément quels sont ces vieux mythes? Le dossier y répond en quatre parties chronologiques. 1. «L'antijudaïsme chrétien », fondateur mais qui perdure du Moyen Âge au $\mathrm{XX}^{\mathrm{e}}$ siècle, 2. "La haine sociale », apparue après la Révolution française qui émancipa les juifs de France, 3. «L'antisémitisme racial » qui trouva son expression la plus aboutie dans la mise en œuvre de la législation du gouvernement de Vichy, 4. « Un nouvel âge ? » interroge la revue en analysant des faits récents.

Première partie :

Un des mythes fondateurs de l'antisémitisme est sans conteste le déicide, accusation lancée d'abord par les Pères de l'Église puis l'Église elle-même qui «affirme son hostilité à l'égard des Juifs dès le Moyen Âge, élaborant une légende noire qui justifie, pour les siècles à venir, brimades et massacres »(Giovanni Miccoli). Le concile du Latran IV de 1215 leur impose le port, sur la poitrine, de la rouelle jaune (ancêtre de l'étoile infligée aux juifs durant la Seconde Guerre mondiale). Les massacres qui accompagnent les croisades, les statues donnant à voir sur les porches des cathédrales une Synagogue aveugle et dépravée en témoignent. Le très chrétien saint Louis, comme d'autres rois de France, lutte contre l'usure en confisquant les biens des juifs et c'est sous son règne que, suite à une controverse publique sur le Talmud, celui-ci est brûlé en 
1241 et en 1244 (Jacques Berlioz). Inquiet pour son salut, le roi adopte une attitude ambiguë faite de condamnation et de relative protection (Marie Dejoux). L'antisémitisme se place alors essentiellement sur le plan religieux, il s'agit d'un antijudaïsme dont se nourrissent des légendes comme la profanation des hosties (analyse d'un tableau par Victor Stoichita). Pourtant l'Espagne dès les XIV ${ }^{\mathrm{e}}-\mathrm{XV}^{\mathrm{e}}$ siècles a «l'obsession de la pureté du sang » et pratique la ségrégation des juifs, annonçant les mesures raciales ultérieures (Jean-Frédéric Schaub). L'antisémitisme religieux continue et le dossier effectue un saut dans le temps en poursuivant avec l'affaire Mortara, en Italie au milieu du $\mathrm{XIX}^{\mathrm{e}}$ siècle durant laquelle l'intransigeance du pape Pie IX, relayée par Veuillot, ferme toute possibilité de négociation : l'enfant enlevé à sa famille juive ne peut être rendu car il est baptisé (Michel Winock). La même intransigeance du Vatican prolonge l'affaire Finaly dans les années 1950 (encart d'André Kaspi). Cette première partie essentiellement centrée sur la période médiévale est accompagnée d'une chronologie s'étendant de 1182 à 14498 (David Nirenberg).

Seconde partie :

Alors que l'émancipation accordée aux juifs par la Révolution a vocation d'en finir avec l'antisémitisme, il n'en est rien. Autorisés à exercer sans restriction tous les métiers, quelques juifs pénètrent dans la société en y gravissant des échelons, c'est alors que s'installe la « haine sociale », même la gauche qui milite pour la démocratie est touchée (Michel Winock). L'ascension des Rothschild (Dominique Borne) entretient la lutte des classes et un Proudhon manifeste violemment son hostilité à la «puissance juive ». Même un Jaurès, d'avant l'affaire Dreyfus, n'est pas choqué par les exactions antijuives commises en Algérie. Il faut la «révolution dreyfusienne» pour faire basculer le socialisme vers le refus de l'antisémitisme ; la présence de Blum, bien qu'exacerbant la haine des juifs, n'est pas étrangère à ce basculement. Actuellement, conclut Michel Winock, la position de la France Insoumise est ambiguë. Dans le même temps, Drumont, "cupide, menteur, volontiers manipulateur », répand son venin avec $L a$ France juive (1886) et son journal La Libre Parole (Grégoire Kauffmann, spécialiste de Drumont), il parvient à fréquenter Hugo, les Goncourt, Flaubert, Maupassant ! Mais «Le moment Dreyfus» vient bousculer ces certitudes chez certains (Michel Winock). Toutefois le revirement ne se fait pas partout: en Algérie (Pierre Michelbach), en Russie (Nicolas Werth) les pogroms sont meurtriers tandis qu'à Vienne l'antisémite Lueger accède à la tête de la municipalité, et la «judéo-maçonnerie » est dénoncée par les antisémites (Judith Kahn).

Troisième partie :

Le développement des sciences biologiques de la fin du $\mathrm{XIX}^{\mathrm{e}}$ siècle et au $\mathrm{XX}^{\mathrm{e}}$ siècle permet l'élaboration d'un « racisme scientifique » que le nazisme reprend à son compte pour définir racialement le juif (Johann Chapoutot). Ainsi, parmi les différentes « races humaines », se distingue le «type juif» opposé au «type aryen» (Pierre-André Taguieff). L'antisémitisme prend sa forme raciale. Dans les années trente, alors que Blum est attaqué physiquement parce que juif, des auteurs comme Céline, Coston, Drieu La Rochelle, Béraud, Brasillach déplorent l'abondance «des nez courbes »! (Michel Winock). Des caricatures dénoncent l'emprise du juif sur le monde, enserrant la planète dans ses bras griffus (Anne-Marie Matard-Bonucci) et les Protocoles des sages de Sion, un faux fabriqué par des Russes, est largement diffusé et connaît des "recyclages infinis » jusqu'à nos jours, dans le monde arabo-musulman et dans l'Europe anciennement communiste (Pierre-André Taguieff). Il en résulte « les années terribles » de 1940 à 1944 qui voient la mise en œuvre de l'extermination des juifs (Annette Wieviorka) et «le meurtre de bureau» quand l'administration de Vichy dénaturalise les juifs devenus français (Claire Zalc). Le gouvernement de Vichy, héritier de ces dérives antisémites, collabore à cette mise à mort : « la police hésite », quelques fonctionnaires refusent cette collaboration (Laurent Joly). Les exemples d'échappatoire 
dont bénéficient trois adolescents en sont la preuve, leur histoire est succinctement évoquée.

Quatrième partie :

«Un nouvel âge » de l'antisémitisme est-il en train de naître ? Après la guerre des Six Jours, le glissement vers l'antisionisme n'est plus à démontrer : " on crie Mort aux Juifs » dans les manifestations antisionistes. Si bien que, de nouveau, l'antisémitisme déborde sur l'extrême gauche, voire la gauche (Pierre-André Taguieff). Dans sa définition, l'antisionisme ne doit pas être confondu avec la critique légitime de la politique du gouvernement israélien (Alain Dieckhoff). En Europe, on constate «le retour aux vieux démons (Annette Wieviorka) tandis que le négationniste de la Shoah, Robert Faurisson, «frappe encore» et se fait acclamer par les admirateurs de Dieudonné (Valérie Igounet). Le 14 mars 2019, l'hebdomadaire polonais Tylko Polska titre : «Comment reconnaître un juif»!

En guise de conclusion à ce dossier, étendu chronologiquement, Dominique Schnapper affirme : «Défendre les juifs, c'est combattre pour la démocratie ». La prolifération de l'antisémitisme met à mal la démocratie rongée sur sa droite et sur sa gauche.

Répondant à une actualité très récente, la rédaction a repris nombre d'articles déjà parus dans des numéros antérieurs, parfois revus et complétés par les auteurs. On peut s'inquiéter de constater que des articles vieux de dix à vingt années n'ont pas vieilli et peuvent de nouveau être livrés à la réflexion sur l'antisémitisme.

Le dossier est enrichi d'une abondante chronologie depuis la destruction du Temple en 70 jusqu'à février 2019, d'un lexique et d'une bibliographie.

Danielle Delmaire

Généalo-J, Revue française de généalogie, $\mathrm{n}^{\circ}$ 137, mars 2019, 55 p.

La revue du Cercle de Généalogie Juive n'est pas un simple bulletin d'informations diffusé entre généalogistes amateurs. Ses contributeurs donnent toujours à lire, à travers des parcours individuels ou familiaux, des articles qui rendent compte de l'histoire des familles juives, ou/et de communautés, et qui nourrissent les connaissances sur l'histoire des juifs en général. Le dernier numéro en est la preuve.

L'historien des communautés juives d'Algérie, Jean Laloum, étudie les «persécutions et déportations [des] Juifs natifs du Constantinois dans la France de Vichy ». Les juifs d'Algérie ne furent pas déportés, néanmoins certains d'entre eux ont connu l'extrême rigueur des camps du sud du pays, mais des noms de juifs originaires d'Algérie figurent sur les listes de déportés car quelques familles s'étaient installées en France métropolitaine avant la guerre et notamment à Marseille où la grande rafle du 22 au 24 janvier 1943 décime la communauté juive. À partir de cas particuliers exhumés de sources archivistiques, l'auteur évoque des parcours semblables et différents à la fois. Deux jeunes gens « maris d'aryenne », deux familles, une vendeuse des quatre saisons sont raflées, parfois sur dénonciation! Le point commun de ces familles raflées : les conjoints « aryens » ou les enfants de « mère aryenne » sont épargnés. Voilà une étude qui dépasse un simple exposé de généalogiste.

Même remarque pour l' " Histoire et mémoire de Fortunée Abignoli » racontée par son arrière-petit-fils, Julien Colet. Le souvenir de Fortunée reste bien flou parmi ses descendants et son arrière-petit-fils dut avoir recours à des archives pour compléter les maigres renseignements que pouvaient lui fournir les membres de sa famille. Il en conclut, à regret, un désir partagé d'oublier les moments douloureux de la guerre, ce qui finit par jeter aux oubliettes un pan de l'histoire familiale et de l'histoire des juifs plus généralement. Car le parcours de Fortunée est original mais fréquent à la fois. Née en Égypte, elle n'y resta pas et s'installa à Marseille après son mariage avec un juif deux 
fois veufs et déjà père. Elle assuma l'éducation de toute cette fratrie alors qu'elle-même devint prématurément veuve. Elle y gagna en indépendance et en autonomie. Elle ne parvint pas à se convaincre du danger et elle fut arrêtée puis emportée vers Auschwitz par le convoi 52. Sa fille Laure, la grand-mère de l'auteur, perdit sa mère, un frère et un compagnon, père de son bébé, qu'elle déclara de mère inconnue pour lui épargner la déportation. Trop de meurtrissures accablaient Laure qui «a transmis à ses filles la souffrance de la Shoah et de la guerre mais non la connaissance » et, plus douloureux encore, «Près de cinquante ans après la Shoah, liens familiaux et religieux étaient brisés » regrette son petit-fils.

De même encore, le récit d'Andrée Margolin, « Une jeune fille juive sous l'Occupation (1940-1944) » publié grâce à son fils, témoigne de la volonté d'une étudiante brillante de mener à son terme ses études. Le récit débute à Paris aux premiers mois de l'Occupation, le père averti et prudent - il a eu connaissance de l'appel du général de Gaulle - est néanmoins arrêté puis interné à Drancy. Ironie tragique : c'est là qu'il reçoit son diplôme d'officier pour services rendus à la France !!! C'est peut-être ce qui lui permet d'être libéré. Commence alors une vie plus errante mais jamais vraiment cachée. Des amis, des voisins, des employeurs les aident et la jeune Andrée parvient coûte que coûte à passer ses examens même si son entrée à l'École Normale Supérieure n'est pas homologuée comme ce fut le cas de tous les candidats juifs. Malgré ses réussites, Andrée constate : «J'ai gardé la vie, j'ai fait mes études presque normalement mais on m'a volé ma jeunesse : je n'ai jamais eu dix-huit ans ».

Plus généalogiste est la contribution d'Anne-Marie Fribourg qui raconte «Une saga américaine : la descendance de Victor Fribourg (Niederwisse, Moselle, 10 janvier 1797 - New York, 7 mai 1884) soldat de l'Empereur et par laquelle on suit brièvement le parcours de chacun de ses neufs enfants et leurs descendants, établis pour la plupart aux USA.

Enfin Éliane Roos-Schul, érudite en épigraphie juive, livre la lecture et l'interprétation d'un « sceau à l'arbre accosté d'oiseaux de Menahem Ezobi » des XV'-XVI ${ }^{\mathrm{e}}$ siècles. 